\title{
Food for thought
}

\author{
Heather B Wood
}

The idea that neurological disease might be prevented or treated through dietary intervention is an attractive one, not least to the manufacturers of nutritional supplements. However, there is limited evidence that such approaches are effective, and they might even be harmful in some cases.

One area that has attracted much attention recently is the relationship between vitamin B and dementia. One study identified a link between low vitamin $B_{12}$ levels and increased brain atrophy in elderly individuals (Vogiatzoglu A et al. [2008] Neurology 71: 826832), and the authors proposed that low vitamin B status could be a modifiable risk factor for cognitive decline. As we report in this month's Research Highlights, Paul Aisen and colleagues at the University of California, San Diego have tested the hypothesis that supplementation with vitamins $B_{6}$ and $B_{12}$ slows cognitive decline in patients with Alzheimer disease (AD). Disappointingly, high-dose vitamin B supplements did not produce significant clinical or cognitive benefits compared with placebo in patients with mild to moderate AD. In addition, the patients who took supplements experienced more adverse effects, including depressive symptoms, than did the controls.

Speaking to WebMD, Aisen concluded, "I do not recommend high-dose vitamins for anybody unless they have a deficiency or an established indication for such treatment. Risk of Alzheimer's disease is not an indication for high-dose B vitamins." Unsurprisingly, Andrew Shao from the Council for Responsible Nutrition, a trade group for the dietary supplement industry, was less pessimistic. He told WebMD, "Vitamins do not have the powerful effects of pharmaceuticals. They are subtle and take a long time to show an effect."

It is important to stress that the patients in the Aisen et al. study had normal vitamin B
"... . We must

remember that

many [nutritional supplements]

have real

pharmacological

effects, not all

of which are

desirable."

HB Wood is the Editor of Nature Clinical Practice Neurology.

Competing interests

The author declared no competing interests.

www.nature.com/clinicalpractice doi:10.1038/ncpneuro0989 levels, and the possibility remains that supplementation could benefit individuals with vitamin B deficiency. In addition, a new line of investigation has been opened by a study showing that vitamin $\mathrm{B}_{3}$ lowers levels of tau protein and improves cognition in a mouse model of AD (Green KN et al. [2008] J Neurosci 28: 11500-11510).

Vitamin D has also been in the news recently. In a second Research Highlight in this issue, we describe a study in which an unusually high prevalence of vitamin $D$ insufficiency was demonstrated in patients with Parkinson disease. This is not the first time that vitamin $D$ deficiency has been implicated in neurological disease; low vitamin $D$ levels have previously been linked to exacerbation of multiple sclerosis and to an increased risk of stroke.

There is a tendency to think of nutritional supplements as harmless at worst and beneficial at best. However, despite what the supplement industry might say, we must remember that many of these compounds have real pharmacological effects, not all of which are desirable. In 2006, Wolfrath et al. reported on the widespread use of nutritional supplements among patients with Parkinson disease (Wolfrath SC et al. [2006] Mov Disord 21: 10981101) and suggested that patients should be warned that certain supplements could interact with drugs already being taken to treat the condition. If the benefits of vitamin supplementation are overstated, there is a danger that some patients might abandon established drug treatments in favor of unproven approaches that are deemed more 'natural'. It is, of course, understandable that patients will want to pursue all available options for the treatment of their condition, but they need to be provided with realistic expectations of the benefits, as well as being informed of any potential risks. 